\title{
The Hydrogen Atomic Model Based on the Electromagnetic Standing Waves and the Periodic Classification of the Elements
}

\author{
Giuseppe Bellotti ${ }^{1}$ \\ ${ }^{1}$ 14/C, S. Gaudenzio Street, 10015 Ivrea (To), Italy \\ Correspondence: Giuseppe Bellotti, 14/C, S. Gaudenzio Street, 10015 Ivrea (To), Italy. E-mail: \\ giuseppe.bellotti@live.it
}

Received: June 19, 2012 Accepted: July 6, 2012 Online Published: July 27, 2012

doi:10.5539/apr.v4n3p141 URL: http://dx.doi.org/10.5539/apr.v4n3p141

\begin{abstract}
By means of a new concept of the Hydrogen atomic structure [where the electron of the Hydrogen is considered not as a non-dimensional object revolving around the atomic nucleus in a field of central forces, but as a three-dimensional electromagnetic spherical standing wave concentrically superimposed on the electromagnetic standing wave of the proton] we can obtain a new periodic classification of the elements. Up till now, chemistry has considered atoms to be made up of sub-shells with 2, 6, 10, 14 electrons and currently, the great number of electrons (14) in sub-shells f creates some problems in the formation of the sixth and seventh periods. Instead in this paper we will only consider sub-shells with 2, 6, 10 electrons, to achieve a better ordering of the heavy metals. Hence, $\mathrm{Ni}, \mathrm{Pd}$, and $\mathrm{Yb}$ have been drawn up in columns in the new periodic classification of elements, and Ytterbium could be proposed as a testing material in the cold fusion experiments. Moreover many new theoretical (albeit unsteady) electromagnetic wave-lengths of Hydrogen Spectrum appear.
\end{abstract}

Keywords: periodic classification of elements, hydrogen atomic model, electromagnetic standing waves, hydrogen spectrum

\section{Introducation}

A new concept of the Hydrogen atomic structure (where the Hydrogen electron is considered not as a non-dimensional object revolving around the atomic nucleus in a field of central forces, but as a three-dimensional electromagnetic (e.m.) spherical standing wave concentrically superimposed on the e.m. standing wave of the proton) has been previously considered in (Bellotti, 2011).

Since the e.m. standing waves have a defined angular frequency $\omega_{\gamma}$, then the wave equation can be written as:

$$
c^{2} \nabla^{2} \psi=\frac{\partial^{2} \psi}{\partial t^{2}}=-\omega_{\gamma}^{2} \psi
$$

where:

$$
\psi(r, \vartheta, \varphi, t)=R(r) \Theta(\vartheta) \Phi(\varphi) T(t)
$$

$\psi$ is a function defined in polar coordinates $(r, \theta, \varphi) ; t$ is time and $c$ is the speed of light.

In (Bellotti, 2011) the following results were demonstrated:

$$
E=\frac{h}{2 \pi} \omega_{\gamma} \propto k^{2}
$$

Where: $E$ is the energy of the e.m. standing wave; $\omega_{\gamma}$ is the angular frequency of the e.m. standing wave and $k=\frac{\pi}{a_{0}}$ is wave number; $a_{0}$ is the radius of the first nodal surface of the electron, considered to be an e.m. spherical standing wave.

$$
\Phi(\varphi)=\frac{1}{\sqrt{\pi}} \sin \left(m \varphi+\frac{\pi}{4}\right)
$$


where $m \in \mathrm{Z}$ is the magnetic quantum number.

The functions $\Theta(\theta)$ are :

$$
\begin{array}{ll}
\Theta(x=\cos (\vartheta))=P_{l}(x)=\frac{1}{2^{l} l !} \frac{d^{l}}{d x^{l}}\left(x^{2}-1\right)^{l} & \text { for } m=0 \\
\Theta(x)=P_{l}^{m}(x)=\left(1-x^{2}\right)^{\frac{|m|}{2}} \frac{d^{|m|}}{d x^{|m|}}\left(P_{l}(x)\right) & \text { for } m \neq 0
\end{array}
$$

The equations in 5 are not normalized. They can be normalized as per (Persico 1971).

$$
T(t)=K \sin \left(\omega_{\gamma} t+G\right)
$$

Where: $K=$ the amplitude $G=$ the phase

$$
\begin{gathered}
R_{0}(r)=\frac{1}{r}\left\{[\sin (k r)]+A_{0}[\cos (k r)]\right\} \\
R_{1}(r)=\frac{1}{r}\left\{\left[\sin (k r)+\frac{\cos (k r)}{k r}\right]+A_{1}\left[\cos (k r)-\frac{\sin (k r)}{k r}\right]\right\} \\
R_{2}(r)=\frac{1}{r}\left\{\left[\sin (k r)+\frac{3 \cos (k r)}{k r}-\frac{3 \sin (k r)}{(k r)^{2}}\right]+A_{2}\left[\cos (k r)-\frac{3 \sin (k r)}{k r}-\frac{3 \cos (k r)}{(k r)^{2}}\right]\right\} \\
R_{3}(r)=\frac{1}{r}\left\{\left[\begin{array}{l}
\left.\sin (k r)+\frac{6 \cos (k r)}{k r}-\frac{15 \sin (k r)}{(k r)^{2}}-\frac{15 \cos (k r)}{(k r)^{3}}\right]+ \\
\left.+A_{3}\left[\cos (k r)-\frac{6 \sin (k r)}{k r}-\frac{15 \cos (k r)}{(k r)^{2}}+\frac{15 \sin (k r)}{(k r)^{3}}\right]\right\}
\end{array}\right.\right. \\
R_{4}(r)=\frac{1}{r}\left\{\begin{array}{l}
{\left[\sin (k r)+\frac{10 \cos (k r)}{k r}-\frac{45 \sin (k r)}{(k r)^{2}}-\frac{105 \cos (k r)}{(k r)^{3}}+\frac{105 \sin (k r)}{(k r)^{4}}\right]+} \\
+A_{4}\left[\cos (k r)-\frac{10 \sin (k r)}{k r}-\frac{45 \cos (k r)}{(k r)^{2}}+\frac{105 \sin (k r)}{(k r)^{3}}+\frac{105 \cos (k r)}{(k r)^{4}}\right]
\end{array}\right\}
\end{gathered}
$$

with the following values of $A_{l}$ ( where $l=0,1,2,3,4$ )

$$
\begin{aligned}
& A_{0}=0 \quad A_{1}=-\frac{1}{\pi}=-0.31830989 \quad A_{2}=-\frac{3 \pi}{\left[\pi^{2}-3\right]}=-1.37195352 \\
& A_{3}=\frac{3\left[2 \pi^{2}-5\right]}{\left[\pi\left(15-\pi^{2}\right)\right]}=2.74343515 \quad A_{4}=\frac{5 \pi\left[21-2 \pi^{2}\right]}{\pi^{4}-45 \pi^{2}+105}=-0.081930363
\end{aligned}
$$

We can verify that for $l=0$ the $R_{0}(r)$ equation meets the boundary condition $a_{0} R_{0}\left(a_{0}\right)=0$ even when the wave number $k=\left(\frac{\pi}{a_{0}}\right)$ is substituted by the wave numbers $k^{*}=j\left(\frac{\pi}{a_{0}}\right)$ with $j \in \mathrm{N}$. The corresponding graphs show that each $a_{0, j}=j a_{0}$ root of $R_{0}(r)$ is shifted to $r=a_{0}$ and we have $\mathrm{n}=j$ nodal spherical surfaces in the radial interval $\left[0, a_{0}\right]$.

\section{The Periodic Classification of the Elements}

Using the symbol ns the sub-shells s joined to the principal quantum number $\mathrm{n}$ can be defined. For every sub-shell ns correspond the functions $R_{0}(j r)=\frac{1}{r} \sin \left(j \frac{\pi}{a_{0}} r\right)$ that vanish for $r=a_{0}$ and that affirm the condition $j=\mathrm{n}$. Then the 
eigenvector $R_{0}(r)$ of the $1 \mathrm{~s}$ sub-shell $(j=1)$ shows one nodal surface within the interval of $r\left[0, a_{0}\right]\left(\right.$ at $\left.r=a_{0}\right)$; the eigenvector $R_{0}(2 r)$ of $2 \mathrm{~s}$ sub-shell $(j=2)$ shows two nodal surfaces within $\left[0, a_{0}\right]$; the eigenvector $R_{0}(3 r)$ of $3 \mathrm{~s}$ sub-shell $(j=3)$, three; and so on. If we establish $a_{0, j}$ to be the $j$ th-root of the function $R_{0}(r)$ shown in equation 7 , then, from (3) and for the ns sub-shells, we can confirm that:

$$
E_{0, j} \propto k^{2}=\left(\frac{\pi}{a_{0}}\right)^{2} \propto\left(j \frac{\pi}{a_{0}}\right)^{2} \propto j^{2} \propto\left(j a_{0}\right)^{2}=a_{0, j}^{2} \quad j=1,2,3,4, \ldots
$$

and, according to equation 21 of (Bellotti, 2009), the total sub-shell energy $E_{l, j}$ that corresponds to each $j$ th-root $a_{l, j}$ of the $R_{l}(r)$ shown in $(7)$ is:

$$
E_{l, j} \propto a_{l, j}^{2}
$$

If all the $w_{l}$ eigenvectors of a given $(l, j)$ sub-shell have the same energy $E_{l, j}{ }^{*}$, then from (10) we have:

$$
\begin{array}{ll}
E_{l, j}^{*}=\frac{E_{l, j}}{w_{l}} \propto \frac{a_{l, j}^{2}}{w_{l}} \Leftrightarrow \sqrt{E_{l, j}^{*}} \propto \frac{a_{l, j}}{\sqrt{w_{l}}} \\
\text { with: } \quad l=0 \quad m=0 \Rightarrow w_{0}=1 \quad \text { sub-shells } \mathrm{s} \\
l=1 \quad m=0, \pm 1 \Rightarrow w_{1}=3 \quad \text { sub-shells } \mathrm{p} \\
l=3 \quad m=0, \pm 1, \pm 2, \pm 3 \Rightarrow w_{3}=7 \quad \text { sub-shells d } \\
l=4 \quad m=0, \pm 1, \pm 2, \pm 3, \pm 4 \Rightarrow w_{4}=9 \quad \text { sub-shells } \mathrm{f}
\end{array}
$$

Where $w_{l}$ (with $l=0,1,2,3, \ldots$ ) are the number of equations present in the sub-shells $l$.

Now by substituting the function $\left(\sqrt{w_{l}} r\right)$ in place of $r$, within the $R_{l}(r)$ shown in (7), we obtain the functions $R_{l}^{*}\left(\sqrt{w_{l}} r\right)$. The roots $a_{l, j}$ of these functions $R_{l}^{*}\left(\sqrt{w_{l}} r\right)$ will be proportional to the square root of the energy $E_{l, j}^{*}$ of each eigenvector $R_{l}(j r)$ that has $j$ roots (or sometimes $j$-1 roots, for $\mathrm{f}$ and g sub-shells) within the interval of $r$ [0, $a_{0}$ ]. The eigenvectors $R_{l}(j r)$ are calculated by substituting $k^{*}=j \pi / a_{0}$ into the equations 7 instead of $k=\pi / a_{0}$; and by imposing the boundary condition $a_{0} R_{l}\left(j a_{0}\right)=0$ in order to calculate the corresponding $A_{l}$ values. Then we can to draw the $R_{l}^{*}\left(\sqrt{w_{l}} r\right)$ functions and obtain the graph shown in Figure 1 where the energies $E_{l, j}^{*}$ of the sub-shells increase in accordance with the following sequence (13):

$$
1 \mathrm{~s}(j=1), 1 \mathrm{p}(j=2), 1 \mathrm{~d}(j=3), 1 \mathrm{p}^{2}(j=3), 1 \mathrm{~s}^{2}(j=2), 1 \mathrm{f}(j=5), 1 \mathrm{~g}(j=6), \ldots
$$

Thus, each shell associated to the principal quantum number $\mathrm{n}$ is formed by the sequence of the sub-shells ns, np, $\mathrm{nd}, \mathrm{np}^{2}, \mathrm{~ns}^{2}, \mathrm{nf}, \ldots$; where $\mathrm{ns}, \mathrm{np}$, nd ... are the first useful root of the functions $R_{0}^{*}\left(\sqrt{w_{0}} r\right), R_{1}^{*}\left(\sqrt{w_{1}} r\right), \ldots$, that belong to the same shell of the principal quantum number n.ns ${ }^{2}, \mathrm{np}^{2}$, etc., are the second useful root of $R_{0}^{*}\left(\sqrt{w_{0}} r\right)$, $R_{1}^{*}\left(\sqrt{w_{1}} r\right), \ldots$, that belong to the same shell of the principal quantum number $\mathrm{n}$. The root of the type $1 \mathrm{~d}^{2}(j=4)$, between $1 \mathrm{p}^{2}$ and $1 \mathrm{~s}^{2}$ in Figure 1 is to be omitted since the sub-shell with minimal energy where there are four nodal surfaces within the interval $\left[0, a_{0}\right]$ has to be the sub-shell $1 \mathrm{f}$. Since the eigenvector $R_{3}(j r)$ of the sub-shell $1 \mathrm{f}$ shows four nodal surfaces within the interval $\left[0, a_{0}\right]$ only when $j=5$, then we have to consider the fifth $\operatorname{root}$ of $R_{3}^{*}\left(\sqrt{w_{3}} r\right)$, whose associated eigenvector is $R_{3}(5 r)$. Now, sub-shell $1 \mathrm{f}$ has an energy greater than the energy of sub-shell $1 \mathrm{~s}^{2}$; then also sub-shell $1 \mathrm{~d}^{2}$ must have an energy greater than the energy of sub-shell $1 \mathrm{~s}^{2}$. Hence, the fourth root of function $R_{2}^{*}\left(\sqrt{w_{2}} r\right)$ does not have any corresponding energy value.

In accordance with the fractals form of the superposition of the e.m. standing waves (Bellotti 2011, 370), we presume a filling up of each shell relative to the principal quantum numbers $n=1,2,3, \ldots$ in accordance with sub-shell sequence (13) and in order to generate Table 1. In Table 1 there are only the s, p, d sub-shells. Therefore, we have to draw a graph (Figure 2) in order that the sub-shells in Table 1 and the roots of the functions $R_{l}^{*}\left(\sqrt{w_{l}} r\right)$ correspond to each other. Now, if we consider the number $j$ of nodal surfaces of the functions $R_{l}(j r)$ (with $l=0,1$, 2) within the interval of $r\left[0, a_{0}\right]$, then this number $j$ also identifies the position of the $j$ th-root $a_{l, j}$ of the corresponding $R_{l}^{*}\left(\sqrt{w_{l}} r\right)$. 
Table 1. First approach to the periodic classification of the elements

\begin{tabular}{ccc}
\hline Principal quantum number & Sub-shells coherent with the filling uporder (13) \\
\hline $\mathrm{n}=1$ & $l=0$ & $1 \mathrm{~s}$ \\
\hline $\mathrm{n}=2$ & $l=0$ & $2 \mathrm{~s}$ \\
& $l=1$ & $2 \mathrm{p}$ \\
\hline $\mathrm{n}=3$ & $l=0$ & $3 \mathrm{~s}$ \\
& $l=1$ & $3 \mathrm{p}$ \\
& $l=2$ & $3 \mathrm{~d}$ \\
\hline $\mathrm{n}=4$ & $l=0$ & $4 \mathrm{~s}$ \\
& $l=1$ & $4 \mathrm{p}$ \\
& $l=2$ & $4 \mathrm{~d}$ \\
& $l=1$ & $4 \mathrm{p}^{2}$ \\
\hline $\mathrm{n}=5$ & $l=0$ & $5 \mathrm{~s}$ \\
& $l=1$ & $5 \mathrm{p}$ \\
& $l=2$ & $5 \mathrm{~d}$ \\
& $l=1$ & $5 \mathrm{p}^{2}$ \\
& $l=0$ & $5 \mathrm{~s}^{2}$ \\
\hline $\mathrm{n}=6$ & $l=0$ & $6 \mathrm{~s}$ \\
& $l=1$ & $6 \mathrm{p}$ \\
& $l=2$ & $6 \mathrm{~d}$ \\
& $l=1$ & $6 \mathrm{p}^{2}$ \\
& $l=0$ & $6 \mathrm{~s}^{2}$ \\
\hline & $l=0$ & $7 \mathrm{~s}$ \\
& $l=1$ & $7 \mathrm{p}$ \\
\hline
\end{tabular}

$$
\begin{aligned}
& R_{l}^{*}\left(\sqrt{w_{l}} r\right) \\
& l=0,1,2,3,4
\end{aligned}
$$

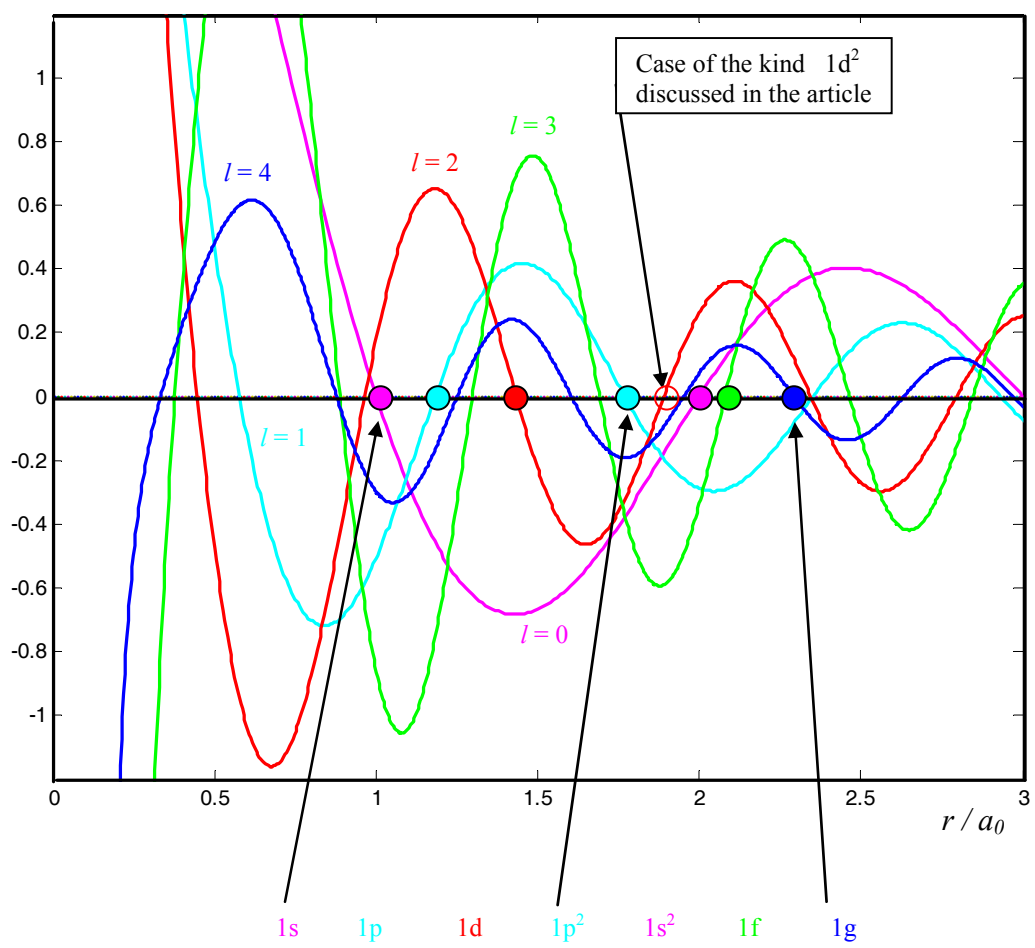

Figure 1. With this graph it is possible to recognize the sequence of sub-shells with increasing energy, valid for each shell that corresponds to the principal quantum number $n$ 
Given that the number of roots within the interval $\left[0, a_{0}\right]$ for each eigenvector $R_{0}(\mathrm{n} r)$ of the sub-shell ns is equal to $\mathrm{n}$, we have to consider all the ordered roots $\mathrm{n}=1,2,3, \ldots$, and 7 of the function $R_{0}^{*}\left(\sqrt{w_{0}} r\right)$. The eighth root of $R_{0}^{*}\left(\sqrt{w_{0}} r\right)$ will be the sub-shell $5 \mathrm{~s}^{2}$, the ninth the sub-shell $6 \mathrm{~s}^{2}$. On the other hand, for each shell related to the principal quantum number $\mathrm{n}$, the number of roots of the functions $R_{I}(j r)$ within the interval [ $\left.0, a_{0}\right]$ for sub-shell $\mathrm{np}(l=1)$ must be equal to $2 \mathrm{n}$; those for sub-shell nd $(l=2)$ must be equal to $3 \mathrm{n}$. For sub-shell $5 \mathrm{~s}^{2}$ we have shown the position of the eighth root that coincides with $8 \mathrm{~s}$; sub-shell $5 \mathrm{p}^{2}$ will have five roots more when compared to $5 \mathrm{~s}^{2}$, (that is 13); sub-shell $4 \mathrm{p}^{2}$ will, instead, have 11 roots [ in fact the hypothetical sub-shell $4 \mathrm{~s}^{2}$ ( that does not exist) coincides with $7 \mathrm{~s}$ and we have to consider $7+4=11$ roots for sub-shell $4 \mathrm{p}^{2}$ ]. Sub-shell $6 \mathrm{~s}^{2}$ coincides with the position 9s and so will have 9 roots; instead sub-shell $6 \mathrm{p}^{2}$ will have six roots more, namely, 15 roots.

Now it is possible to generate Table 2, where to each $j$ th-root of the functions $R_{0}^{*}\left(\sqrt{w_{0}} r\right), R_{1}^{*}\left(\sqrt{w_{1}} r\right)$ and $R_{2}^{*}\left(\sqrt{w_{2}} r\right)$ corresponds an eigenvector $R_{0}(j r), R_{1}(j r)$ and $R_{2}(j r)$; moreover each eigenvector $R_{0}(j r), R_{1}(j r)$ and $R_{2}(j r)$ within the interval $\left[0, a_{0}\right]$ gives the number $j$ of nodal surfaces indicated (as roots) in Table II, and all these numbers $j$ of roots coincide with the $j$ th-root $a_{l, j}$ position of the functions $R_{l}^{*}\left(\sqrt{w_{l}} r\right)$. Now we can order the sub-shells shown in Table 2 according to the increasing energies $E_{l, j}{ }^{*}$ shown in Figure 2 and begin building Table III. Through tests, we can check that, for Hydrogen atom, the frequencies $v_{A, B}$ that correspond to the transitions $B$ $\rightarrow A$ [ where the status $A$ is characterized by the angular quantum number $l_{A}$ and by the $j_{A}$ position of the considered root of the function $R_{l_{1}^{*}}^{*}\left(\sqrt{w_{l_{A}}} r\right)$; and status $B$ is characterized by the angular quantum number $l_{B}$ and by the $j_{B}$ position of the considered root of the function $R_{l_{B}}^{*}\left(\sqrt{w_{l_{B}}} r\right)$ ] are obtained in the following Formula 14 (White, 1934):

$$
v_{A, B}=\frac{E_{B}^{*}-E_{A}^{*}}{h}=R_{\infty} c\left(\frac{1}{n_{A}^{2}}-\frac{1}{n_{B}^{2}}\right)=3.28984(23) 10^{15}\left(\frac{1}{n_{A}^{2}}-\frac{1}{n_{B}^{2}}\right) \mathrm{Hz}
$$

where:

$$
R_{\infty}=\text { Rydberg constant }=1.09737312(11) 10^{7} \mathrm{~m}^{-1}
$$

Table 2. Second approach to the periodic classification of the elements. The $j$ number of roots within the $r$

\begin{tabular}{|c|c|c|c|c|}
\hline \multirow{2}{*}{$\begin{array}{l}\text { Principal quantum number } \\
\qquad \mathrm{n}=1\end{array}$} & \multicolumn{4}{|c|}{ Sub-shells coherent with the filling up order (13) } \\
\hline & $l=0$ & $1 \mathrm{~s}$ & 1 & root \\
\hline \multirow[t]{2}{*}{$\mathrm{n}=2$} & $l=0$ & $2 \mathrm{~s}$ & 2 & roots \\
\hline & $l=1$ & $2 \mathrm{p}$ & 4 & roots \\
\hline \multirow[t]{3}{*}{$\mathrm{n}=3$} & $l=0$ & $3 \mathrm{~s}$ & 3 & roots \\
\hline & $l=1$ & $3 p$ & 6 & roots \\
\hline & $l=2$ & $3 d$ & 9 & roots \\
\hline \multirow[t]{4}{*}{$\mathrm{n}=4$} & $l=0$ & $4 \mathrm{~s}$ & 4 & roots \\
\hline & $l=1$ & $4 p$ & 8 & roots \\
\hline & $l=2$ & $4 d$ & 12 & roots \\
\hline & $l=1$ & $4 p^{2}$ & 11 & roots \\
\hline \multirow[t]{5}{*}{$\mathrm{n}=5$} & $l=0$ & $5 \mathrm{~s}$ & 5 & roots \\
\hline & $l=1$ & $5 \mathrm{p}$ & 10 & roots \\
\hline & $l=2$ & $5 \mathrm{~d}$ & 15 & roots \\
\hline & $l=1$ & $5 p^{2}$ & 13 & roots \\
\hline & $l=0$ & $5 s^{2}$ & 8 & roots \\
\hline \multirow[t]{5}{*}{$\mathrm{n}=6$} & $l=0$ & $6 s$ & 6 & roots \\
\hline & $l=1$ & $6 p$ & 12 & roots \\
\hline & $l=2$ & $6 d$ & 18 & roots \\
\hline & $l=1$ & $6 \mathrm{p}^{2}$ & 15 & roots \\
\hline & $l=0$ & $6 s^{2}$ & 9 & roots \\
\hline \multirow[t]{2}{*}{$\mathrm{n}=7$} & $l=0$ & $7 \mathrm{~s}$ & 7 & roots \\
\hline & $l=1$ & $7 p$ & 14 & roots \\
\hline
\end{tabular}
interval $\left[0, a_{0}\right]$ of the $R_{l}(j r)$ eigenvector coincides with the $j$ th-root position of the $R_{l}^{*}\left(\sqrt{w_{l}} r\right)$ function 
All the values of the Physical constants are taken from (Jordan, 1988).

$n_{A}{ }^{2}, n_{B}{ }^{2}=$ numerical data (shown in Table 3) proportional to the square of the corresponding root values of the functions $R_{l_{A}}^{*}\left(\sqrt{w_{l_{A}}} r\right)$ and $R_{l_{B}}^{*}\left(\sqrt{w_{l_{B}}} r\right)$,

where we have considered the minimum value of $n_{A}=n_{l=0, j=1}=1$

Equation (14) is in accordance with the following equation (15), that was obtained from (9), (10) and (11), applied to ns sub-shells $(l=0)$ :

$$
E_{0, j}^{*}=\frac{E_{0, j}}{w_{0}} \propto \frac{k^{2}}{w_{0}} \propto\left(\frac{\pi}{a_{0}}\right)^{2} \propto \frac{1}{\left(j a_{0}\right)^{2}}=\frac{1}{a_{0, j}^{2}} \propto-\frac{1}{n_{0, j}^{2}} \quad j=1,2,3,4, \ldots
$$

where $n_{0, j}{ }^{2}$ has the same meaning as $n_{A}^{2}, n_{B}^{2}$ in (14), for $l=0$.

Now we can assume that the sub-shell energy $E_{l, j}^{*}$, that corresponds to each $j$ th-root $a_{l, j}$ of $R_{l}^{*}\left(\sqrt{w_{l}} r\right)$ obtained from (7), is:

$$
E_{l, j}^{*}=\frac{E_{l, j}}{w_{l}} \propto \frac{1}{a_{l, j}^{2}} \propto-\frac{1}{n_{l, j}^{2}}
$$

where the $n_{l, j}{ }^{2}$ in (16) correspond to either the $n_{A}{ }^{2}$ or $n_{B}{ }^{2}$ in (14), according to the $\left(l_{A}, j_{A}\right)$ or $\left(l_{B}, j_{B}\right)$ values. Both the $n_{A}{ }^{2}$ or $n_{B}{ }^{2}$ values are proportional to the square of the corresponding root values $a_{l_{A}, j_{A}}, a_{l_{B}, j_{B}}$ of the functions $R_{l_{A}}^{*}\left(\sqrt{w_{l_{A}}} r\right)$ and $R_{l_{B}}^{*}\left(\sqrt{w_{l_{B}}} r\right)$.

Now, by putting $n_{A}{ }^{2}=1$ and $n_{B}{ }^{2} \rightarrow \infty$ into (14), we can calculate the maximum energy $\left|E_{A}{ }^{*}\right|=R_{\infty} c h$ that in a $B \rightarrow$ $A$ transition can be emitted as a light spectrum row; this energy is half the energy $\mathrm{E}=\alpha^{2} m_{e} c^{2}$ of an elementary e.m. standing wave of radius $a_{0}$ (Bellotti, 2011). On the other hand this result is also valid for all the s, p, $\mathrm{d}$ sub-shells. In fact we can substitute the equivalent energy referred to the last column of Table 3 , in place of $n_{A}^{2}$, in 14; and we can substitute a value tending towards infinity in place of $n_{B}^{2}$; and in accordance with (16) (and its marking), we can obtain two possibilities for emitting a light ray for each eigenvector (an e.m. standing wave) that forms each sub-shell. In fact:

$$
-E_{l, j}^{*}=R_{\infty} \operatorname{ch} \frac{1}{n_{l, j}^{2}}=\frac{1}{2}\left(\alpha^{2} m_{e} c^{2}\right) \frac{1}{n_{l, j}^{2}}
$$

Where: $h=$ Planck constant; $\alpha=$ fine structure constant and $m_{e}=$ electron mass.

By returning to (12) and the definition of $w_{l}$ (with $l=0,1,2,3, \ldots$ ) being the equation number present in each sub-shell $l$, we can assume that in the sub-shells s $(l=0)$ we have $w_{0}=2$ possibilities of light emissions (and this value of $w_{0}$ can replace the number of equations $\left.w_{0}=1\right)$. In a similar way, we can obtain $w_{1}=6$ instead of $w_{1}=3$ for the sub-shells $\mathrm{p}(l=1) ; w_{2}=10$ for the sub-shells $\mathrm{d}(l=2)$; and so on. The energy property of this eigenvector brings to mind the Pauli principle. So the sequence of the sub-shells shown in Table 3 faithfully reproduces the properties of the elements in their periodic classification in a natural way. Now we can build Table 4. Up till now, chemistry has considered atoms to be made up of sub-shells with 2, 6, 10,14 electrons and, currently, the great number of electrons, 14 , in sub-shells $\mathrm{f}$ creates some problems in the formation of the sixth and seventh periods. Instead, Table 3 and Table 4 show that we can consider only sub-shells with 2, 6, 10 electrons, to achieve a better ordering of the heavy metals. Hence, $\mathrm{Ni}, \mathrm{Pd}$, and $\mathrm{Yb}$ have been drawn up in columns in the new periodic classification of elements, and Ytterbium could be proposed as an optimum material for cold fusion.

The last column of Table 3 shows some real numbers. This is in accordance with Milan Perkovac's considerations on equations (100) - (104) of (Perkovac, 2010). It is possible to go deeper into Perkovac's model in (Perkovac, 2002; Perkovac, 2003). 
Table 3. Sequence of the sub-shells filling up, according to the increase in energy

\begin{tabular}{|c|c|c|c|c|}
\hline Sub-shells & $\begin{array}{c}\text { Transitions } \\
\text { Number }\end{array}$ & $\begin{array}{l}\text { Atomic Numbers } \\
\text { of the Elements } \\
\text { in each sub-shell }\end{array}$ & $\begin{array}{l}\text { Elements } \\
\text { of the } \\
\text { sub-shell }\end{array}$ & $\begin{array}{c}\text { Energy } n_{A}^{2} \propto a_{l, j}^{2} \\
\text { of the } B=\infty \rightarrow A \\
\text { Transition }\end{array}$ \\
\hline $1 \mathrm{~s}$ & 2 & $Z=1-2$ & $\mathrm{H} \mathrm{He}$ & 1.000000 \\
\hline $2 \mathrm{~s}$ & 2 & $Z=3-4$ & $\mathrm{Li} \mathrm{Be}$ & 4.000000 \\
\hline $2 p$ & 6 & $Z=5-10$ & $\begin{array}{l}\text { B C N } \\
\text { O F Ne }\end{array}$ & 5.530507 \\
\hline $3 \mathrm{~s}$ & 2 & $Z=11-12$ & $\mathrm{Na} \mathrm{Mg}$ & 9.000000 \\
\hline $3 p$ & 6 & $Z=13-18$ & $\begin{array}{l}\text { Al Si P } \\
\mathrm{S} \mathrm{Cl} \mathrm{Ar}\end{array}$ & 12.32801 \\
\hline $4 \mathrm{~s}$ & 2 & $Z=19-20$ & $\mathrm{~K} \mathrm{Ca}$ & 16.00000 \\
\hline $3 d$ & 10 & $Z=21-30$ & $\begin{array}{l}\mathrm{Sc} \mathrm{Ti} \text { V Cr Mn } \\
\text { Fe Co Ni Cu Zn }\end{array}$ & 17.17438 \\
\hline $4 p$ & 6 & $Z=31-36$ & $\begin{array}{l}\mathrm{Ga} \mathrm{Ge} \mathrm{As} \\
\mathrm{Se} \mathrm{Br} \mathrm{Kr}\end{array}$ & 21.79214 \\
\hline $5 \mathrm{~s}$ & 2 & $Z=37-38$ & $\mathrm{Rb} \mathrm{Sr}$ & 25.00000 \\
\hline $4 d$ & 10 & $Z=39-48$ & $\begin{array}{l}\text { Y Zr Nb Mo Tc } \\
\text { Ru Rh Pd Ag Cd }\end{array}$ & 30.13389 \\
\hline $5 p$ & 6 & $Z=49-54$ & $\begin{array}{l}\text { In } \mathrm{Sn} \mathrm{Sb} \\
\mathrm{Te} \mathrm{I} \mathrm{Xe}\end{array}$ & 33.92294 \\
\hline $6 \mathrm{~s}$ & 2 & $Z=55-56$ & $\mathrm{Cs} \mathrm{Ba}$ & 36.00000 \\
\hline $4 p^{2}$ & 6 & $Z=57-62$ & $\begin{array}{l}\mathrm{La} \mathrm{Ce} \mathrm{Pr} \\
\mathrm{Nd} \mathrm{Pm} \mathrm{Sm}\end{array}$ & 40.98833 \\
\hline $5 \mathrm{~d}$ & 10 & $Z=63-72$ & $\begin{array}{l}\text { Eu Gd Tb Dy Ho } \\
\text { Er Tm Yb Lu Hf }\end{array}$ & 46.69335 \\
\hline $6 \mathrm{p}$ & 6 & $Z=73-78$ & $\begin{array}{l}\text { Ta W Re } \\
\text { Os Ir Pt }\end{array}$ & 48.72039 \\
\hline $7 \mathrm{~s}$ & 2 & $Z=79-80$ & $\mathrm{Au} \mathrm{Hg}$ & 49.00000 \\
\hline $5 p^{2}$ & 6 & $Z=81-86$ & $\begin{array}{l}\mathrm{Tl} \mathrm{Pb} \mathrm{Bi} \\
\text { Po At Rn }\end{array}$ & 57.11912 \\
\hline $5 s^{2}$ & 2 & $Z=87-88$ & Fr Ra & 64.00000 \\
\hline $7 \mathrm{p}$ & 6 & $Z=89-94$ & $\begin{array}{l}\text { Ac Th Pa } \\
\text { U Np Pu }\end{array}$ & 66.18450 \\
\hline $6 \mathrm{~d}$ & 10 & $Z=95-104$ & $\begin{array}{l}\text { Am Cm Bk Cf Es } \\
\text { Fm Md No Lw Ku }\end{array}$ & 66.85278 \\
\hline $6 p^{2}$ & 6 & $Z=105-110$ & $\mathrm{Ha} \ldots$ & 75.91658 \\
\hline $6 s^{2}$ & 2 & $Z=111-112$ & $\ldots$ & 81.00000 \\
\hline
\end{tabular}


Table 4. New periodic classification of the elements. It is interesting to notice the blue column-sequence Ni Pd $\mathrm{Yb}(\mathrm{No})$ and to consider Ytterbium for cold fusion experiments

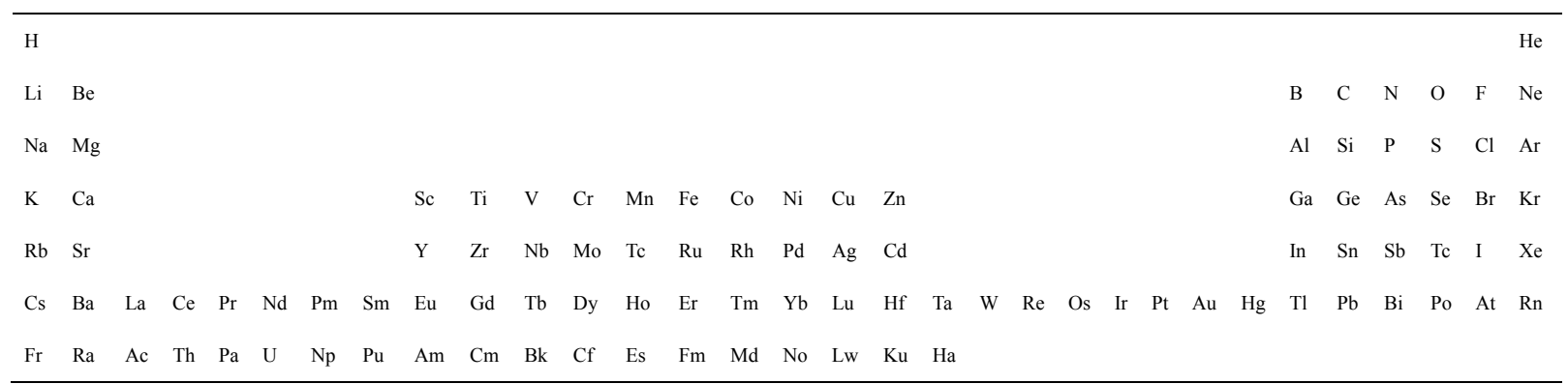

\section{Calculation of the Spectral Wave Lengths of Hydrogen}

Now we can calculate all the wave-lengths of the Hydrogen spectrum by using the following formula (18) of first approximation, derived from (14) (White, 1934):

$$
\lambda_{A, B}=\frac{10^{10}}{R_{\infty}}\left(\frac{n_{A}^{2} n_{B}^{2}}{n_{B}^{2}-n_{A}^{2}}\right)
$$

where: $R_{\infty}=1.09737312(11) 10^{7} \mathrm{~m}^{-1}$.

$n_{A}{ }^{2}, n_{B}{ }^{2}$ are the values shown in the last column of Table 3 .

They correspond to the $B \rightarrow A$ transition.

Table 5 shows the most important wavelength that could form the Hydrogen spectrum. But in equation (18) a greater number of Hydrogen spectrum rows were computed compared to the fundamental ones obtained in Quantum Mechanics. This result has to be confirmed by means of a dynamic experimental check. In fact, in all induced mechanical vibrations, the contribution of high order eigenvectors vanishes quickly and only the main eigenvectors, that correspond to the spectrum rows joined to $l=0$ ( $\mathrm{s} \mathrm{sub}$-shells), are present in the stationary state. As in Quantum Mechanics, now the whole values of $\mathrm{n}_{A}{ }^{2}$ (see Table 3) correspond to $l=0$. Hence, we can obtain the normal rows of emission spectrum of Hydrogen by equation (18). On the other hand, spectrum rows joined to $l=1$ ( $\mathrm{p}$ sub-shells) and to $l=2$ ( $\mathrm{d}$ sub-shells), that correspond to the non-whole $\mathrm{n}_{A}{ }^{2}$ values, are present only in the transient of Hydrogen atoms excitation. But the presence of either a transient or a stationary state can only be justified when it is possible to consider each Hydrogen atom as a continuous function of energy in a space domain; in other words: when each Hydrogen atom can be considered as an e.m. standing wave. And this condition complies to the fundamental hypothesis of this article. Of course this approach allows for the quantization of energy, since the wave equation eigenvectors can take on different discrete values of energy (see Figure 2).

Then it seems that the traditional idea, i.e. the rows of atomic emission spectra are produced by quantum jumps of the electrons between two different energy levels, has to be formulated in a different way.

Table 5. The most important rows of Hydrogen spectrum expressed in $\mathrm{nm}$

\begin{tabular}{llll}
$6 \mathrm{~s}^{2} 1 \mathrm{~s} 92.26579$ & $6 \mathrm{~d} 1 \mathrm{~s} \quad 92.5105$ & $6 \mathrm{p}^{2} 1 \mathrm{~s} 92.34309$ \\
$7 \mathrm{p} 1 \mathrm{~s}$ & 92.52469 & $5 \mathrm{p}^{2} 1 \mathrm{~s} 92.75052$ & $5 \mathrm{~s}^{2} 1 \mathrm{~s} 92.57317$ \\
$7 \mathrm{~s} 1 \mathrm{~s}$ & 93.02518 & $5 \mathrm{~d} 1 \mathrm{~s} \quad 93.12102$ & $6 \mathrm{p} 1 \mathrm{~s} \quad 93.03631$ \\
$4 \mathrm{p}^{2} 1 \mathrm{~s} 93.40554$ & $5 \mathrm{p} 1 \mathrm{~s} \quad 93.89459$ & $6 \mathrm{~s} 1 \mathrm{~s} \quad 93.73034$ \\
$4 \mathrm{~d} 1 \mathrm{~s}$ & 94.25456 & $4 \mathrm{p} 1 \mathrm{~s} \quad 95.50946$ & $5 \mathrm{~s} 1 \mathrm{~s} \quad 94.92365$ \\
\hline
\end{tabular}




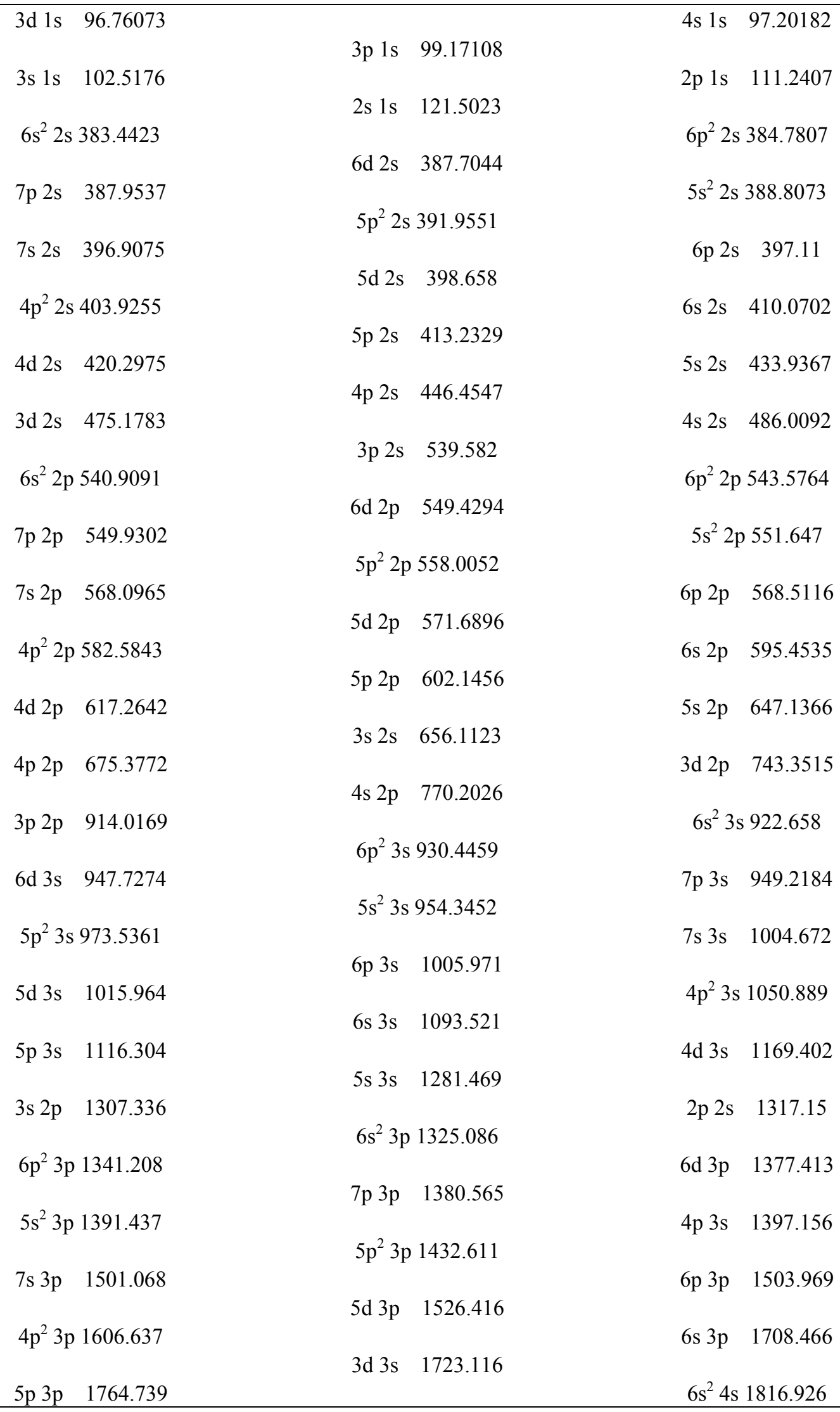




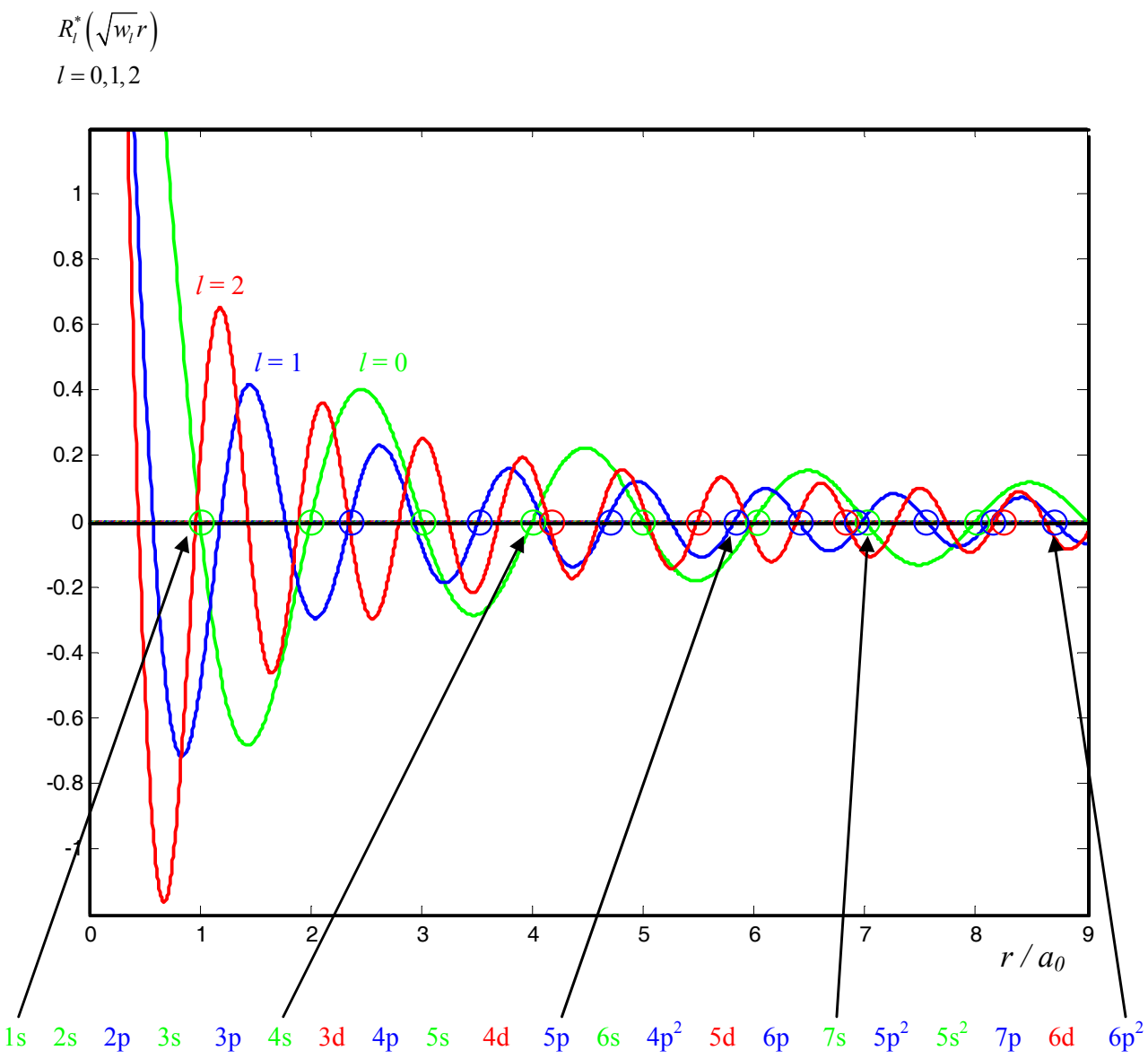

Figure 2. Sequence according to the increase in energy in the sub-shells filling up, in order to reproduce the Periodic Classification of the Elements

\section{Conclusions}

In this paper, we have considered the electron of Hydrogen as a three-dimensional e.m. spherical standing wave concentrically superimposed onto the e.m. standing wave of the proton. By hypothesizing a fractals form for the Hydrogen structure, it was possible to obtain a new periodic classification of the elements. Up till now, chemistry has considered atoms to be made up of sub-shells with 2, 6, 10,14 electrons and, currently, the great number of electrons, 14 , in sub-shells $f$ creates some problems in the formation of the sixth and seventh periods. Instead, in this paper we considered only sub-shells with 2, 6, 10 electrons, to achieve a better ordering of the heavy metals. Then $\mathrm{Ni}, \mathrm{Pd}$, and $\mathrm{Yb}$ have been drawn up in columns in the new periodic classification of the elements. So it is possible to propose Ytterbium for the cold fusion experiments. Moreover, in accordance with Milan Perkovac's analysis (Perkovac, 2010), we obtained many fast decaying new theoretical e.m. wave-lengths of Hydrogen Spectrum. Do any appropriate tests exist for validating this? If so, the proposed approach to Hydrogen atomic structure (a superposition of the e.m. spherical standing waves of electron and proton) will have made a worthy experimental contribution. Furthermore, it is interesting to note that equations (7) are solutions of either the wave equation (1) (being applied to the Hydrogen atom) or the dynamic bi-Laplacian equation (Bellotti, 2009) that analyses the Hydrogen nuclear properties. Could this mathematical link be used in establishing a theory of cold fusion?

\section{References}

Bellotti, G. (2009). The dynamic bi-Laplacian equation in polar coordinates and the magic numbers of atomic nucleus. Phys. Essays, 22(268). http://dx.doi.org/10.4006/1.3141024

Bellotti, G. (2011). The hydrogen atomic model founded on the electromagnetic standing waves. Phys. Essays, 24(364). http://dx.doi.org/10.4006/1.3601519 
Jordan, E. C. (1985). Reference Data for Engineers (VII - 1988). Indianapolis, IN: Howard W. Sams \& Company. 3.13-16.

Perkovac, M. (2002). Quantization in Classical Electrodynamics. Phys. Essays, 15(41). http://dx.doi.org/10.4006/1.3025509

Perkovac, M. (2003). Absorption and Emission of Radiation by an Atomic Oscillator. Phys. Essays, 16(162). http://dx.doi.org/10.4006/1.3025572

Perkovac, M. (2010). Statistical test of Duane-Hunt's law and its comparison with an alternative law. Retrieved from arXiv:1010.6083v1

Persico, E. (1939). Fondamenti della Meccanica Atomica (VI-1971). Bologna: Zanichelli. 216- 223.

White, H. E. (1934). Introduction to Atomic Spectra. New York, NY: Mc Graw-Hill - Kögakusha. 